\title{
Diabetes mellitus y riesgo cardiovascular: recomendaciones del Grupo de Trabajo Diabetes y Enfermedad Cardiovascular de la Sociedad Española de Diabetes (SED, 2015)
}

\section{Francisco Arrieta ${ }^{a, *}$, Pedro Iglesias ${ }^{b}$, Juan Pedro-Botet ${ }^{c}$, Francisco Javier Tébar ${ }^{d}$, Emilio Ortega ${ }^{\mathrm{e}}$, Andreu Nubiola ${ }^{\mathrm{f}}$, Jose Luis Pardog ${ }^{\mathrm{g}}$, Gonzálo Fernando Maldonado ${ }^{\mathrm{h}}$, Juan Carlos Obaya ${ }^{i}$, Pablo Matute ${ }^{j}$, Romina Petrecca ${ }^{k}$, Nuria Alonso', Elena Sarabia ${ }^{m}$, Victor Sánchez-Margalet ${ }^{n}$, José Juan Alemán ${ }^{n}$, Jorge Navarro ${ }^{\circ}$, Antonio \\ Becerra $^{\mathrm{b}}$, Santiago Duran ${ }^{\mathrm{p}}$, Manuel Aguilar ${ }^{\mathrm{q}}$, Fernando Escobar-Jiménez ${ }^{\mathrm{r}}$ y Grupo de Trabajo Diabetes y Enfermedad Cardiovascular de la Sociedad Española de Diabetes (SED)}

a Unidad Enfermedades Metabólicas del Adulto (CSUR), Servicio de Endocrinología y Nutrición Hospital Universitario Ramón y Cajal, IRYCIS, CIBEROBN, Madrid, España

b Servicio de Endocrinología y Nutrición Hospital Universitario Ramón y Cajal, IRYCIS, Madrid, España

c Unidad de Lípidos y Riesgo Vascular, Servicio de Endocrinología y Nutrición, Parc de Salut Mar, Barcelona, España

d Servicio de Endocrinología y Nutrición, Hospital Universitario Virgen de la Arrixaca, Murcia, España

e Servicio de Endocrinología y Nutrición, Hospital Clínic, IDIBAPS, CIBEROBN, Barcelona, España

f Servicio Endocrinologia, Hospital LÉsperit Sant, Sta. Coloma Gramenet, Barcelona, España

s Centro de Salud Orihuela I, Orihuela (Alicante), Alicante, España

h Servicio de Endocrinología y Nutrición, Hospital Universitario Araba, Vitoria-Gasteiz, España

' Centro de Salud Chopera, Madrid, España

j Instituto Médico del Puerto de Santamaría, Cádiz, España

k Servicio de Endocrinología y Nutrición, Hospital Universitario de La Princesa, Madrid, España

' Servicio de Endocrinología y Nutrición, Hospital Universitari Germans Trias i Pujol, Badalona, España

m Profesora del Grado en Ciencias de la Actividad Física y del Deporte en Cardenal Spínola CEU, Sevilla

n Servicio Bioquímica Clínica, Hospital Universitario Virgen Macarena, Sevilla, España

ñ Centro de Salud de Tacoronte, Tenerife, España

- Dirección Atención Primaria, IIS INCLIVA, CIBERESP, Valencia, España

P Unidad de Gestión Clínica de Endocrinología y Nutrición, Hospital Universitario Ntra. Sra. de Valme. Sevilla, España

q Servicio de Endocrinología y Nutrición, Hospital Puerta del Mar, Cádiz, España

r Servicio de Endocrinología y Nutrición Hospital Universitario San Cecilio, Granada, España

\footnotetext{
De acuerdo con los autores y editores este artículo se publica de forma íntegra también en: Clin Invest Arterioscl. 2015;27(4):181-192. * Autor para correspondencia.

Correo electrónico: arri68@hotmail.com (F. Arrieta).
} 


\section{PALABRAS CLAVE}

Guía práctica clínica; Factores de riesgo cardiovascular; Diabetes mellitus

\section{KEYWORDS}

Clinical practice guidelines;

Cardiovascular risk

factors;

Diabetes mellitus
Resumen El presente documento actualiza las recomendaciones de práctica clínica del manejo de los factores de riesgo cardiovascular (FRCV) en la diabetes mellitus (DM). Es un consenso médico realizado por un panel de expertos independiente de la Sociedad Española de Diabetes (SED). Se han propuesto y actualizado varios consensos de diferentes sociedades científicas o médicas con el fin de mejorar los resultados terapéuticos. La valoración del RCV en la población general puede carecer de sensibilidad para la evaluación individual en determinados grupos de riesgo como los diabéticos. Se revisan los factores de riesgo tradicionales y no tradicionales, así como las estrategias de intervención para el control de los FRCV en los pacientes diabéticos como la dieta, el control ponderal, el ejercicio físico, los hábitos tóxicos, el control glucémico, tensional y lipídico, así como la antiagregación plaquetaria. Confiamos en que estas pautas ayuden a los médicos en la toma de decisiones en su actividad asistencial. Se expone una actualización de los conceptos más relevantes y de mayor interés clínico-práctico $y$, a su vez realista, para reducir el RCV de los diabéticos como se venía haciendo regularmente por parte del Grupo de Enfermedad Cardiovascular de la SED.

(C) 2015 Publicado por Elsevier España, S.L.U. Este es un artículo Open Access bajo la licencia CC BY-NC-ND (http://creativecommons.org/licenses/by-nc-nd/4.0/).

Diabetes mellitus and cardiovascular risk: Working group recommendations of Diabetes and Cardiovascular Disease of the Spanish Society of Diabetes (SED, 2015)

\begin{abstract}
The present paper updates the Clinical Practice Recommendations for the management of cardiovascular risk factors (CVRF) in diabetes mellitus. This is a medical consensus agreed by an independent panel of experts from the Spanish Society of Diabetes (SED). Several consensuses have been proposed by scientific and medical Societies to achieve clinical goals. However, the risk score for general population may lack sensitivity for individual assessment or for particular groups at risk, such as diabetics. Traditional risk factors together with nontraditional factors are reviewed throughout this paper. Intervention strategies for managing CVRF in the diabetic patient are reviewed in detail: balanced food intake, weight reduction, physical exercise, smoking cessation, reduction in $\mathrm{HbA1c}$, therapy for high blood pressure, obesity, lipid disorders, and platelet anti-aggregation. It is hoped that these guidelines can help clinicians in the decisions of their clinical activity. This regular update by the SED Cardiovascular Disease Group of the most relevant concepts, and of greater practical and realistic clinical interest, is presented in order to reduce CVR of diabetics.

(c) 2015 Published by Elsevier España, S.L.U. This is an open access article under the CC BY-NC-ND license (http://creativecommons.org/licenses/by-nc-nd/4.0/).
\end{abstract}

\section{Introducción}

Se entiende como factor de riesgo cardiovascular (FRCV) aquella característica biológica, condición y/o modificación del estilo de vida que aumenta la probabilidad de padecer o de fallecer por cualquier causa de una enfermedad cardiovascular (ECV) en aquellos individuos que lo presentan a medio y largo plazo. Para la evaluación de este riesgo se han elaborado tablas o ecuaciones de probabilidades basadas en estudios prospectivos poblacionales. Estas tablas se aplican posteriormente a los individuos de la población a la que van dirigidas para su análisis estratificado y pormenorizado. Se consideran fundamentalmente los llamados FRCV clásicos (edad, sexo, tabaquismo, diabetes, colesterol total, colesterol ligado a lipoproteínas de baja densidad [CLDL], colesterol ligado a lipoproteínas de alta densidad [CHDL] y niveles de presión arterial [PA]). Otros FRCV llamados no clásicos (historia familiar, obesidad, distribución de la grasa corporal, trigliceridemia, estrés, nivel socioeconómico, etc.) pueden servir para modular el riesgo calculado. El RCV global obtenido de esta manera marca las acciones preventivas y los objetivos de control de algunos de los FRCV modificables en ellas incluidos. En ocasiones, a pesar de las estrategias preventivas, tanto del estilo de vida como farmacológicas, no es posible reducir los niveles de un determinado FRCV a unos objetivos adecuados. En estos casos debemos actuar sobre los otros FRCV modificables para disminuir el RCV global.

Son múltiples las guías clínicas que se han encargado de evaluar los diferentes FRCV, establecer objetivos de tratamiento y servir de referencia para profesionales y sociedades médicas ${ }^{1-4}$. La finalidad última de estas guías es la prevención (primordial, primaria o secundaria) de la ECV. Intentan además proporcionar homogeneidad bajo criterios de equidad en el sistema sanitario, teniendo en cuenta las limitaciones en la utilización de recursos, hacer predecible los procesos y el coste y, por último, permitir la planificación de recursos por los efectos esperados sobre los pacientes y la sociedad $^{5}$. La existencia de estas guías no garantiza su aplicación en la práctica clínica por los profesionales médicos por diversas razones ${ }^{6,7}$. 
Como hemos indicado, las tablas de riesgo tienen como objetivo la prevención de la ECV en la población general. Una de sus limitaciones es que son imprecisas para la evaluación del riesgo individual o de ciertos grupos de especial riesgo. Entre estos grupos, en el área de nuestra especialidad destacan las personas con diabetes y/o con otras formas familiares de dislipidemia, en especial la hipercolesterolemia familiar o la hiperlipidemia familiar combinada. En relación con los sujetos con diabetes las principales sociedades científicas (American Diabetes Association [ADA] y European Association for the Study of Diabetes [EASD]) establecen un riesgo a priori alto o muy alto, y por ello recomiendan utilizar objetivos preventivos concretos ${ }^{3,8}$. Sin embargo, el control integral de los mismos se alcanza en menos del $10-15 \%$ de los pacientes ${ }^{9,10}$. En el presente trabajo revisamos los aspectos más relevantes de los últimos 5 años en relación con los FRCV y la diabetes y, tal y como se venía haciendo regularmente ${ }^{1,2}$, presentamos las recomendaciones del Grupo de Trabajo Diabetes y Riesgo Cardiovascualr de la Sociedad Española de Diabetes (SED) (tabla 1).

\section{Modificación del estilo de vida: dieta, ejercicio físico y tabaquismo}

Uno de los estudios de más relevancia por su diseño, duración y población analizada que preconizaba la importancia de intervenir sobre el estilo de vida fue el Look $A H E A D^{11}$. Sin embargo, los resultados de esta intervención, basada a grandes rasgos en la reducción ponderal mediante actividad física y dieta hipocalórica «pobre» en grasa, no han sido todo lo óptimos que se esperaba, como señalan resultados publicados recientemente tras 10 años de seguimiento ${ }^{12-14}$. La modificación del estilo de vida es importante, especialmente en aquellos individuos con mayor índice de masa corporal (IMC) $y / o$ índice cintura/cadera. Por otro lado, también es importante la detección precoz de la desnutrición y/o riesgo de padecerla cuando el paciente con diabetes es anciano, por el mayor riesgo de presentar sarcopenia asociada. Para ello, disponemos de medios de despistaje sencillos como el Malnutrition Universal Screening Tool (MUST) ${ }^{15}$ o informatizados como el Control Nutricional (CONUT) ${ }^{16}$. Además, para todos los individuos con sobrepeso u obesidad, y por tanto en riesgo de desarrollar diabetes, se recomienda la pérdida de peso por su efecto beneficioso, entre otros factores, sobre la insulinorresistencia. Para conseguir una adecuada pérdida ponderal es importante conocer la ingesta calórica del paciente y recomendar una dieta que facilite la pérdida del $5-10 \%$ de peso, siendo en estos momentos más reforzada tras los resultados del estudio Look $A H E A D$ y del estudio PREDIMED (equilibrada pero sin pérdida de peso). La dieta mediterránea se puede presentar como el paradigma de dieta cardioprotectora y por tanto recomendable ${ }^{17}$. Si esta misma dieta, discretamente hipocalórica y asociada a actividad física moderada, es incluso mejor y segura en términos de protección cardiovascular, es una de las preguntas a las que intentará responder el estudio PREDIMEDPLUS, actualmente en desarrollo (www.predimedplus.com). La dieta mediterránea se caracteriza por un alto consumo en frutas y verduras, cereales y legumbres, frutos secos y aceite de oliva, todos de óptima calidad, consumo moderado de pescado, aves y huevos a ser posible de corral, y una baja
Tabla 1 Recomendaciones del Grupo de diabetes y enfermedad cardiovascular (SED, 2014-2015)
1. Modificación del estilo de vida: dieta, ejercicio físico y tabaquismo
Dieta cardiosaludable: dieta mediterránea
Ejercicio físico aeróbico regular al menos
$150 \mathrm{~min} /$ semana de actividad física aeróbica de intensidad moderada (50-70\% de la frecuencia cardíaca máxima), repartidas en al menos 3 días de la semana evitando estar más de 2 días consecutivos sin ejercicio
Abandono del hábito tabáquico

2. Obesidad

Si sobrepeso/obesidad leve: pérdida ponderal entre $5-10 \%$

Indicaciones cirugía bariátrica puede considerarse en adultos con DM2 e IMC $\geq 35 \mathrm{~kg} / \mathrm{m}^{2}$, especialmente si la DM2 o las comorbilidades asociadas son difíciles de controlar con un estilo de vida cardiosaludable y/o tratamiento farmacológico

3. Control glucémico $\mathrm{HbA} 1 \mathrm{c}<7 \%$

$\mathrm{HbA} 1 \mathrm{c} 7-8 \%$, o incluso > 8\%, en pacientes con ECV, especialmente si edad avanzada, antecedentes de hipoglucemia grave, esperanza de vida limitada, enfermedad microvascular avanzada o complicaciones macrovasculares, con diabetes de larga duración

\section{Lípidos-estatinas (ver tabla 2)}

Objetivo clásico niveles de cLDL en prevención primaria $<100 \mathrm{mg} / \mathrm{dl}$; prevención secundaria $<70$ $\mathrm{mg} / \mathrm{dl}$

\section{HTA}

Presión arterial $<140 / 90 \mathrm{~mm} \mathrm{Hg}$

Si nefropatía: IECA primera elección

Segundo escalón ARA II, antagonistas del calcio, diuréticos

Otros: bloqueadores beta, bloqueadores alfa, antagonistas de la aldosterona y agentes de acción central

6. Acido acetil salicílico (AAS)

Prevención primaria: dosis bajas (100 mg/día) si $\mathrm{RCV}>10 \%$ a los 10 años

Prevención secundaria: dosis bajas (100 mg/día)

\section{Vitaminas}

Vitamina $D>30 \mathrm{ng} / \mathrm{ml}$ (valorar tratamiento farmacológico por debajo de 30 según edad, época de año, niveles previos. Tratar con suplementación farmacológica con niveles por debajo de $20 \mathrm{ng} / \mathrm{ml}$ ) Monitorizar vitamina $B_{12}$, especialmente si está en tratamiento con metformina

ingesta de productos lácteos, carnes rojas, carnes procesadas y dulces, además de la ingesta de vino en moderación consumido con las comidas. En líneas generales, esta se ha demostrado efectiva no solo la prevención cardiovascular, 
sino también en el control de las complicaciones asociadas a la diabetes ${ }^{18,19}$.

A día de hoy a las personas en riesgo de padecer diabetes mellitus tipo 2 (DM2) se les aconseja seguir las recomendaciones del Departamento de Agricultura de los Estados Unidos (USDA). En este sentido se propone consumir fibra en la dieta $(14 \mathrm{~g}$ de fibra $/ 1.000 \mathrm{kcal})$ y alimentos con granos integrales (la mitad de la ingesta de granos). Se debe limitar el consumo de bebidas azucaradas. La ingesta de grasas saturadas debe corresponder $a<7 \%$ del total de las calorías ingeridas y se contraindica la ingesta de grasas trans y se apoya el estilo de ingesta derivado de la dieta mediterránea $^{3}$. Si los adultos con diabetes optan por consumir alcohol el consumo debe ser moderado (limitar su consumo a una una bebida al día o menos para las mujeres adultas y 2 bebidas al día o menos para los hombres adultos). No se recomienda en la población diabética el suplemento sistemático de antioxidantes como las vitaminas E, c y caroteno debido a la falta de evidencia científica sobre su eficacia clínica y a la preocupación de su seguridad a largo plazo. Se recomienda planificar las comidas incluyendo la optimización de la elección de alimentos para satisfacer la cantidad diaria recomendada de todos los micronutrientes. Es interesante conocer la cantidad de hidratos de carbono de los alimentos, y saber elegir los alimentos según sea su carga glucémica y/o índice glucémico ${ }^{20-22}$. Es muy importante el aporte calórico y su distribución a lo largo del día para evitar el sobrepeso al aumentar las calorías y favorecer las hiperglucemias posprandiales. Por último, señalar la necesidad de una correcta hidratación, siendo el agua y las infusiones las mejores fuentes. Para ello, es importante advertir a los pacientes que las bebidas edulcoradas «no reemplazan» el agua y deben limitarse.

La importancia del ejercicio físico fue señalada en 1992 por la American Heart Association (AHA), que incluyó la inactividad física como un factor de riesgo coronario, asociándose esta también con el riesgo de $\mathrm{DM}^{23-25}$. El ejercicio físico regular aumenta la captación de glucosa, disminuye el riesgo de DM2 y previene la hipertensión arterial, siendo el efecto más acusado en hipertensos, con una disminución media de 6-7 mm $\mathrm{Hg}$ en la PAS y PAD. Otro de los beneficios del ejercicio es el aumento del cHDL. Clásicamente se aconsejaba que el paciente con diabetes bien controlado debía realizar ejercicio aeróbico de intensidad moderada $(50-70 \%$ de la frecuencia cardíaca máxima: 220 menos la edad en años), dependiendo de la situación basal de cada persona durante al menos 30 min y como mínimo 5 días a la semana ${ }^{26}$. Hoy en día se recomienda que los diabéticos realicen al menos $150 \mathrm{~min} / \mathrm{semana}$ de actividad física aeróbica de intensidad moderada $\mathbf{5 0 - 7 0 \%}$ de la frecuencia cardíaca máxima), repartidas en al menos 3 días de la semana evitando estar más de 2 días consecutivos sin ejercicio $^{27-30}$. Se debe valorar el tipo de actividad y ejercicio físico, la frecuencia, la duración e intensidad del ejercicio, especialmente en el anciano ${ }^{30}$. Se debe advertir a los diabéticos en tratamiento con insulina que presentan hipoglucemia no reconocida o episodios de hipoglucemia grave de la disminución de la resistencia a la insulina de los tejidos periféricos con mayor captación de glucosa, como el músculo, y por tanto disminución de los niveles plasmáticos de glucosa (favoreciendo el riesgo de hipoglucemia), para lo cual debemos no solo controlar las glucemias capilares antes y durante el ejercicio, sino realizar un control especialmente postejercicio con el fin de evitar las hipoglucemias tardías asociadas a la actividad física.

El tabaco es el principal FRCV modificable. Recientemente, un metanálisis señaló que los diabéticos fumadores tenían un incremento significativo del RCV total, mortalidad, ictus e infarto de miocardio comparado con los no fumadores ${ }^{31,32}$. En la práctica diaria debemos aconsejar a todos los pacientes que dejen de fumar o usar productos derivados del tabaco. Hay que recurrir, con la aceptación del paciente para dejar de fumar, a unidades específicas de deshabituación tabáquica y/o prescribir tratamiento para ello como componente rutinario dentro del cuidado del paciente diabético.

\section{Obesidad mórbida: papel de la cirugía bariátrica en la diabetes mellitus tipo 2}

La obesidad es definida por la Organización Mundial de la Salud (1998) como una enfermedad epidémica que constituye un problema de salud, en la que una acumulación anormal o excesiva de grasa perjudica la salud y el bienestar $^{33}$. Si bien la obesidad es una enfermedad multifactorial de etiología diversa, y en ocasiones incierta, cabe destacar la influencia de 2 tipos de factores: ambientales o modificables y factores genéticos, endógenos o no modificables. El objetivo inicial del tratamiento de la obesidad es la reducción del porcentaje de grasa corporal hasta unos niveles en los que mejoren los factores de riesgo asociados, reduciendo las complicaciones y, por ello, mejorando la calidad de vida. Numerosos estudios han demostrado que un incremento de grasa corporal conlleva un aumento concomitante de $\mathrm{FRCV}^{34}$. El riesgo relativo de diabetes en los varones con un IMC de $35 \mathrm{~kg} / \mathrm{m}^{2}$ es 40 veces superior al de aquellos con un IMC de $23 \mathrm{~kg} / \mathrm{m}^{2}$. Pequeñas pérdidas de peso del $5-10 \%$ se corresponden con un mejor control, no solo de parámetros clínicos y metabólicos, sino también psicológicos, y todo ello sin necesidad de apoyo farmacológico, solo mediante cambios en el estilo de vida y modificaciones dietéticas ${ }^{35}$.

La cirugía bariátrica (CB), como ya se señalaba en $2009^{1,2}$, puede considerarse en adultos con $D M 2$ e $I M C \geq 35 \mathrm{~kg} / \mathrm{m}^{2}$, especialmente si la DM2 o las comorbilidades asociadas son difíciles de controlar con un estilo de vida cardiosaludable y/o tratamiento farmacológico. Es primordial tener en cuenta al valorar los costes que los individuos con DM2 que se someten a CB necesitan adicionalmente modificaciones del «estilo de vida» de por vida, y monitorización médica continuada y estricta. Estudios con un tamaño muestral reducido realizado en pacientes con IMC entre 30 y $35 \mathrm{~kg} / \mathrm{m}^{2}$ y $\mathrm{DM}^{36}$ han demostrado que la CB sigue presentando beneficios glucémicos; sin embargo, estos resultados preliminares no deben ser generalizados. Los beneficios a largo plazo, la relación coste eficacia y los riesgos de la CB en el tratamiento de la DM2 deben ser evaluados en ensayos bien diseñados y controlados con tratamiento médico óptimo, y actuando terapéuticamente sobre el estilo de vida como grupo control. 
La CB, a través de las diferentes técnicas quirúrgicas, ya sean restrictivas, malabsortivas o mixtas, es una medida eficaz para la pérdida ponderal en los obesos graves, y las guías clínicas apoyan su indicación en pacientes con $\mathrm{IMC}>35 \mathrm{~kg} / \mathrm{m}^{2}$ y DM2 $2^{3,36-42}$.

La CB ha demostrado la casi o completa normalización de la glucemia en el $40-95 \%$ de los pacientes con DM2, dependiendo del tiempo de evolución de la diabetes, del procedimiento quirúrgico elegido, de la capacidad de reserva pancreática y de los criterios de remisión utilizados $^{39}$. La remisión de la DM2 tiende a ser menor en aquellos procedimientos que solo restringen la capacidad gástrica, y es mayor en los que realizan un bypass gastroyeyunal. Los procedimientos que incluyen bypass intestinal pueden tener efectos metabólicos que son independientes de sus efectos sobre el peso, posiblemente por su efecto a nivel del sistema incretínico ${ }^{43}$. La CB mejora los FRCV y, a largo plazo, los episodios cardiovasculares en la DM2 ${ }^{44,45}$. Las desventajas de la CB se relacionan directamente con el elevado coste y los riesgos asociados, además del «abandono y pérdida» de los pacientes en la mayoría de las series. En este sentido, la morbimortalidad directamente relacionada con la CB ha disminuido en los últimos años, siendo a los 30 días del $0,28 \%$, similar a la descrita para una colecistectomía laparoscópica. Las complicaciones a largo plazo incluyen deficiencias de vitaminas y minerales ${ }^{44,46}$, osteoporosis $y$, más raramente, hipoglucemia, a menudo grave, derivada de la hipersecreción de insulina, debiéndose descartar la nesidioblastosis ${ }^{47}$.

En resumen, la CB está indicada en los adultos con un $I M C \geq 35 \mathrm{~kg} / \mathrm{m}^{2}$ y $D M 2$, especialmente si la diabetes 0 las comorbilidades asociadas son difíciles de controlar con tratamiento médico. Actualmente no hay suficiente evidencia para recomendar la CB en los pacientes con DM2 e $I M C \leq 35 \mathrm{~kg} / \mathrm{m}^{2}$. Los análisis retrospectivos y estudios actuales sugieren que la CB puede ser coste eficaz en la $\mathrm{DM} 2^{37,48-50}$.

\section{Control glucémico: hemoglobina glucosilada y riesgo cardiovascular}

Después de la modificación de los niveles de glucemia basal en ayunas (GA), considerando cifras $\geq 126 \mathrm{mg} / \mathrm{dl}(7 \mathrm{mmol} / \mathrm{l})$ como criterio diagnóstico de DM2, y aceptando como ayuno la no ingesta calórica durante al menos $8 \mathrm{~h}$, hay que destacar la introducción de la hemoglobina glucosilada (HbA1c) como nueva herramienta diagnóstica de $\mathrm{DM} 2^{48}$. Así, se han establecido niveles de $\mathrm{HbA} 1 \mathrm{c} \geq 6,5 \%$ como criterio diagnóstico de DM, siempre y cuando se realice en un laboratorio que utilice un método estandarizado según el National Glycohemoglobin Standarization Program (NGSP), certificado y estandarizado para el Diabetes Control and Complications Trial, International Diabetes Federation y la EASD y el paciente no tenga anemia o hemoglobinopatía que alteren los niveles de $\mathrm{HbA}_{1} \mathrm{c}^{51-53}$. Se deben considerar las preferencias del paciente para seleccionar adecuadamente entre las alternativas diagnósticas, qué prueba diagnóstica de diabetes usaremos, basándonos en las circunstancias del paciente, como son la inconveniencia de la obtención de muestras de ayuno y el índice de sospecha clínica ${ }^{53}$.
Diferentes estudios han confirmado la importancia del control glucémico en la $D M 2^{54-56}$. Sabemos que una disminución de la $\mathrm{HbA} 1 \mathrm{c}$ del $0,9 \%$ reduce los episodios cardiovasculares en torno al 10-15\%. La disminución de la $\mathrm{HbA} 1 \mathrm{C}$ a cifras cercanas al $7 \%$ reduce las complicaciones microangiopáticas y macrovasculares ${ }^{56}$. Por lo tanto, una meta razonable es una concentración de $\mathrm{HbA} 1 \mathrm{c} \sim 7 \%$. Hoy en día se aconseja individualizar el grado de control glucémico, así en el paciente adulto joven sin otros FRCV y sin complicaciones se debería aspirar a unos objetivos de control glucémico más estrictos, utilizando fármacos más potentes para alcanzar unos objetivos más precisos de HbA1C $(\sim 6,5 \%)$ para pacientes seleccionados, siempre que se consiga sin hipoglucemias significativas $u$ otros efectos adversos. En pacientes con ECV, especialmente los de edad avanzada, como señalamos más adelante, antecedentes de hipoglucemia grave, esperanza de vida limitada, enfermedad microvascular avanzada o complicaciones macrovasculares, con diabetes de larga duración, el objetivo de la $\mathrm{HbA} 1 \mathrm{c}$ podría ser menos exigente, entre $7 \%$ y $8 \%$ o incluso superior ${ }^{57}$.

Es evidente que los pacientes con DM1 deben ser tratados con múltiples dosis de insulina (3-4 dosis diarias) o infusión subcutánea continua de insulina, debiéndose elegir los análogos de insulina para reducir el riesgo de hipoglucemia. Sin embargo, en la DM2, si no hay contraindicación y es tolerada, la metformina es el tratamiento de elección inicial ${ }^{58,59}$. Es importante recordar que la metformina es el único hipoglucemiante oral que se asocia a un menor riesgo de ECV. Cuando la monoterapia con un agente no insulínico a las dosis máximas toleradas no alcanza o no mantiene el objetivo terapéutico de $\mathrm{HbA} 1 \mathrm{c}$ en un tiempo de 3 a 6 meses como máximo, se debe añadir un segundo fármaco oral, agonistas del receptor GLP-1 o insulina individualizando para cada individuo analizando sus comorbilidades y/o complicaciones secundarias de la diabetes asociada ${ }^{58}$.

En el momento de la elección del fármaco debemos considerar la eficacia, el coste, el perfil de seguridad, efectos sobre el peso, las comorbilidades, el riesgo de hipoglucemia, la hiperglucemia posprandial asociada con la resistencia periférica a la insulina y alteración de la fase precoz y tardía de secreción de insulina y la hiperglucemia basal, caracterizada por la resistencia hepática a la insulina y alteración de la fase primera y de secreción de insulina, así como las preferencias del paciente. Todo ello favorecerá la adherencia al tratamiento con la disminución de la morbimortalidad, reduciendo los elevados costes de las complicaciones de la diabetes ${ }^{5,60-63}$.

\section{Presión arterial: control de la hipertensión arterial en la diabetes mellitus tipo 2}

Las nuevas guías europeas de prevención cardiovascular ${ }^{64-67}$ refuerzan las recomendaciones de las guías europeas de HTA de 2009 sin aportar ningún dato novedoso relevante. Se recomienda medir la PA del paciente diabético en cada visita. Si se detectan cifras elevadas se debe realizar el despistaje pertinente. El objetivo de control debe individualizarse, aconsejando de forma global una PAS $<140 \mathrm{~mm} \mathrm{Hg}$. Cifras más bajas de PAS, por debajo de $130 \mathrm{~mm} \mathrm{Hg}$, pueden ser apropiadas en individuos más jóvenes y en pacientes 
con microalbuminuria. El objetivo de PAD en los diabéticos es $<90 \mathrm{~mm} \mathrm{Hg}$, si bien el límite no está bien establecido, aceptándose entre $80-90 \mathrm{~mm} \mathrm{Hg}$ según la edad y comorbilidades asociadas ${ }^{66,67}$.

Los pacientes con PA confirmada $\geq 140 / 90 \mathrm{~mm} \mathrm{Hg}$, además de realizar cambios en el estilo de vida, pérdida de peso (cuando hay sobrepeso), seguir los consejos de la Dietary Approaches Stop Hypertension (cuando hay hipertensión ${ }^{68,69}$, que incluye la reducción del sodio y el aumento de la ingesta de potasio), la reducción del consumo de alcohol y el aumento de la actividad física, debe iniciarse una terapia farmacológica si no se logran los objetivos de control tensional. Los pacientes con diabetes e HTA deben ser tratados farmacológicamente con un inhibidor de la enzima convertidora de angiotensina (IECA) o un antagonista de los receptores de angiotensina (ARA ॥), por su mayor efecto protector contra la aparición o la progresión de nefropatía. Ante la intolerancia a uno de ellos puede sustituirse por el otro. Para alcanzar los objetivos terapéuticos generalmente se requiere la combinación de 2 o más fármacos en su dosis máxima. Es importante administrar uno o más antihipertensivos antes de acostarse con el fin de evitar la HTA nocturna, al constituir la PA nocturna un predictor de episodio cardiovascular más importante que la PA diurna ${ }^{68}$. No se recomienda el uso de la terapia combinada de IECA con ARA II, en particular en pacientes con nefropatía diabética. En los casos en los que se considerase imprescindible, debe llevarse a cabo bajo la supervisión de un especialista, con una estrecha monitorización de la función renal, balance hidroelectrolítico y PA. La combinación de aliskiren con IECA o ARA ॥ en pacientes con alteración de la función renal o diabetes está contraindicada. El candesartán y valsartán se mantienen autorizados para el tratamiento de la insuficiencia cardiaca en combinación con un IECA únicamente en aquellos pacientes que no pueden utilizar antagonistas de los mineralcorticoides.

Si se utilizan IECA, ARA ॥ o diuréticos hay que monitorizar la función renal y el nivel de potasio sérico. Cuando la PA es $>140 / 90 \mathrm{~mm} \mathrm{Hg}$ podrá añadirse un antagonista del calcio $y$, si persiste el mal control, un betabloqueante. En las embarazadas con diabetes e HTA se sugiere un objetivo de PA de 110-129/65-79 mm Hg para evitar el deterioro de la salud materna a largo plazo y minimizar daños en el crecimiento fetal. Los IECA y los ARA ॥ están contraindicados durante el embarazo. Es importante determinar la albuminuria una vez al año en los DM1 con una duración de la diabetes $\geq 5$ años, y en todos los DM2 en el momento del diagnóstico. Las últimas guías han recomendado como objetivo terapéutico en pacientes con DM2 un valor $\leq 140 / 90 \mathrm{~mm} \mathrm{Hg}$. Por lo tanto, el control tensional y la prevención de la morbilidad y la mortalidad relacionada es claramente alcanzable $e^{3,66,67}$. Sin embargo, el conocimiento, tratamiento y control de la HTA son persistentemente bajos en todo el mundo ${ }^{70}$. Uno de los grandes desafíos es evitar la inercia terapéutica (dejando a los pacientes diabéticos con valores de PA de $140 / 90 \mathrm{~mm} \mathrm{Hg}$ o superior), ya que esto daría lugar a una carga inaceptable en términos de vidas humanas, secuelas y costes socioeconómicos. Es importante que los médicos responsables participen activamente en el reto de mejorar el porcentaje de pacientes con PA controlada, máxime cuando en los pacientes hipertensos diabéticos es especialmente difícil alcanzar los objetivos de control tensional con monoterapia hipotensora. La gran mayoría de estos pacientes requiere la combinación de 2 o más fármacos. El primer componente de dicha combinación debe ser un fármaco que bloquee el sistema renina-angiotensina, preferiblemente un IECA, y si no es tolerado elegir un ARA ॥. La ADA aconseja tanto un diurético (hidroclorotiazida o clortalidona) como un antagonista del calcio como segundo fármaco antihipertensivo. En pacientes con un filtrado glomerular $(F G)<30 \mathrm{ml} / \mathrm{min} / 1,73$ $\mathrm{m}^{2}$, debe sustituirse el diurético tiazídico por un diurético de asa. Muchos diabéticos precisan de 3 o más fármacos, utilizándose los bloqueadores beta, bloqueadores alfa, antagonistas de la aldosterona y agentes de acción central. Por último, recordar que debemos controlar la PA nocturna, siendo necesario ajustar la dosificación/distribución de los fármacos y controlar la adherencia al tratamiento farmacológico.

\section{Hiperlipidemia/hipercolesterolemia: ¿dosis fijas de estatinas o niveles de colesterol ligado a lipoproteínas de baja densidad?}

La dislipidemia es un factor patogénico clave en el desarrollo y en la progresión de la lesión vascular. Las ecuaciones de cohortes pueden utilizarse para estimar el RCV a los 10 años tanto en diabéticos como en no diabéticos. Existen en Internet calculadoras on-line para facilitar y conocer el RCV, así como la incorporación de programas informáticos de historia clínica que incluyen calculadoras que automáticamente proporcionan el riesgo coronario en prevención primaria ${ }^{70}$. Es primordial determinar en los pacientes con DM2 el perfil lipídico en ayunas, al menos anualmente, con el fin de valorar y plantear objetivos terapéuticos con mayor precisión. En los adultos con un perfil lipídico dentro de los rangos deseables (cLDL $<100 \mathrm{mg} / \mathrm{dl}, \mathrm{cHDL}>50 \mathrm{mg} / \mathrm{dl}$ y triglicéridos $<150 \mathrm{mg} / \mathrm{dl}$ ), la evaluación de los lípidos puede repetirse cada 2 años (ADA).

Para mejorar el perfil lipídico de los diabéticos se recomienda modificar el estilo de vida para reducir el consumo de grasas saturadas, grasas trans y colesterol; aumentar el consumo de ácidos grasos $\omega-3$, sobre todo en personas que no ingieren habitualmente pescados, fibra viscosa y estanoles/esteroles vegetales; la pérdida de peso (si procede) y aumentar la actividad física. Independientemente del perfil lipídico basal y de la modificación del estilo de vida, deben recibir tratamiento con estatinas a dosis adecuada según los objetivos recomendados, en todos los diabéticos con ECV establecida ${ }^{71-73}$.

Particularmente, en pacientes con DM2, es necesaria la prevención precoz e intensiva mediante tratamiento hipolipidemiante independiente de las concentraciones basales de cLDL, y con objetivos más bajos para el perfil lipídico (tablas 1 y 2). Para pacientes con DM2 y ECV o enfermedad renal crónica (ERC) y que tienen uno o más de los demás FRCV, el valor óptimo de CLDL debe ser $<70 \mathrm{mg} / \mathrm{dl}^{64}$.

La terapia con estatinas es eficaz para la reducción de episodios cardiovasculares. Sin embargo, estudios recientes señalan que las estatinas también confieren un mayor riesgo de desarrollar diabetes y hay que vigilar la densitometría ósea en estos pacientes ${ }^{74,75}$. Todas las estatinas se asocian 
Tabla 2 Tratamiento con estatinas según objetivos de reducción de colesterol ligado a lipoproteínas de baja densidad en diabéticos tipo 2

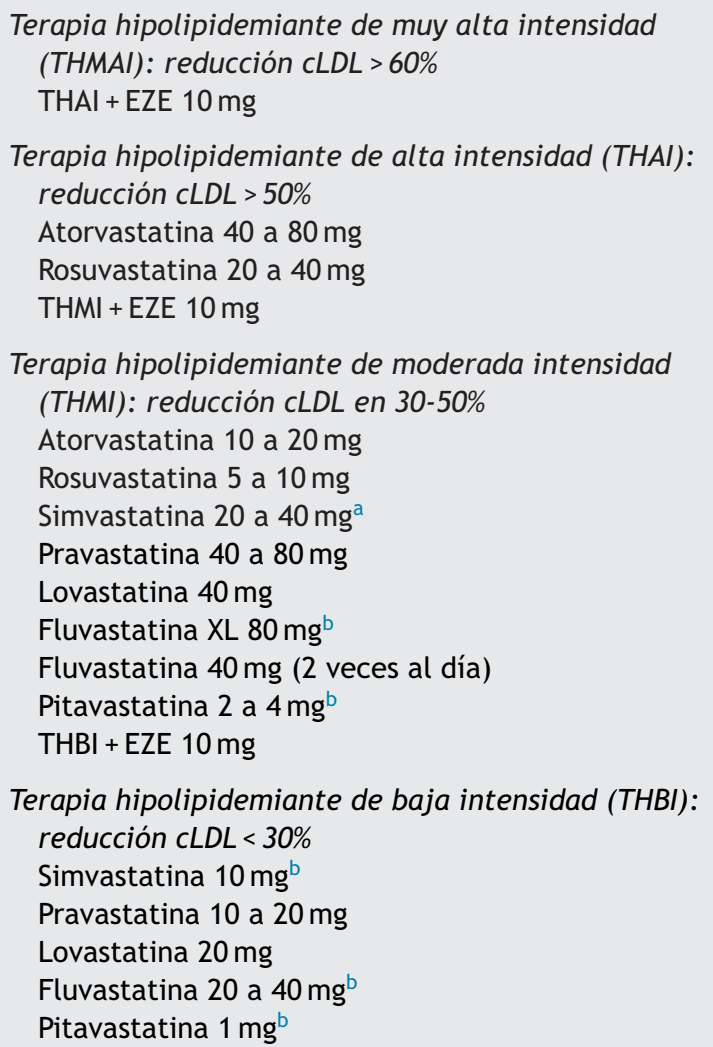

Terapia hipolipidemiante de baja intensidad (THBI): reducción $C L D L<30 \%$

Diabéticos con CLDL $70-190 \mathrm{mg} / \mathrm{dl}$ iniciar terapia de moderada intensidad. En diabéticos $<400>75$ años evaluar riesgo beneficio y preferencias, es decir: la evidencia no aclara el uso de estatina en este último grupo de pacientes.

a Contraindicadas dosis más altas por la incidencia de efectos adversos.

b Aprobados para uso clínico por la Food and Drug Administration, pero no testados en estudios controlados aleatorios.

en mayor o menor grado con un modesto incremento en el riesgo de $\mathrm{DM} 2$ incidente que aumenta con la edad, la presencia de componentes del síndrome metabólico, así como la potencia y dosis de la estatina. El estudio JUPITER en prevención primaria puso de manifiesto los beneficios cardiovasculares y de la evolución de la mortalidad después del tratamiento reglado con estatinas, que fueron superiores al riesgo de la diabetes, incluso entre los que estaban en mayor riesgo de desarrollar $\mathrm{DM} 2^{69}$. Las estatinas, por tanto, siguen siendo la principal herramienta para modificar el RCV debido a la reducción en la concentración de cLDL, pese a su potencial efecto diabetogénico, existiendo diferencias entre las estatinas ${ }^{74-76}$. Según la ADA las estatinas se recomiendan en los pacientes con DM2 sin ECV, con $>40$ años con uno o más FRCV (antecedentes familiares de ECV, HTA, tabaquismo, dislipidemia o albuminuria).

En las recomendaciones de los diferentes National Cholesterol Education Program-Adult Treatment Panel (NCEP ATP $)^{77}$ el objetivo terapéutico eran las concentraciones de
CLDL. Sin embargo, la reciente guía del American College of Cardiology (ACC)/AHA, publicada en $2013^{76}$, ha dado lugar a múltiples consideraciones con diferentes puntos de vista de distintas sociedades científicas por el nuevo paradigma de tratamiento con estatinas, abandonando los objetivos terapéuticos en $\mathrm{CLDL}^{78,79}$. Para los pacientes con menor riesgo que los anteriores (por ejemplo sin ECV manifiesta y $<40$ años), pero cuyo cLDL es $>100 \mathrm{mg} / \mathrm{dl}$ o múltiples FRCV, se debía considerar el tratamiento con estatinas, además de la modificación del estilo de vida. El ACC/AHA señala que los diabéticos con niveles de cLDL entre $70-190 \mathrm{mg} / \mathrm{dl}$ deben iniciar terapia de moderada intensidad, basándose no en los niveles de cLDL como objetivo, sino en la tolerancia a las estatinas y efecto a dosis fijas según el RCV (tabla 1). Se recomienda iniciar el tratamiento de alta intensidad si el $\mathrm{RCV}>7,5 \%$. En caso de tener menos de 40 o más de 75 años, se debe evaluar el riesgo beneficio y las preferencias del paciente, dada la importancia de la adherencia al tratamiento, es decir, la evidencia no aclara el uso de estatinas en este último grupo de pacientes. Es importante destacar que se ha cambiado el paradigma de los objetivos terapéuticos de colesterol como se venía aconsejando, es decir, $\mathrm{cLDL}<70 \mathrm{mg} / \mathrm{dl} \mathrm{y}<100 \mathrm{mg} / \mathrm{dl}$ para prevención secundaria y primaria, respectivamente, y niveles de $\mathrm{cHDL}>40 \mathrm{mg} / \mathrm{dl}$, en función de estudios recientes que señalan que el beneficio se obtiene con el uso de estatinas a la dosis máxima tolerada por el paciente, habiéndose visto en los grupos que muestran beneficio con el tratamiento de estatinas, y esto no depende de los niveles de colesterol alcanzados (tabla 2). Tampoco se ha encontrado un beneficio en añadir otro tipo de fármacos hipolipidemiantes para alcanzar las metas de colesterol no HDL, reducir la apolipoproteína B, la lipoproteína(a) y los triglicéridos ni aumentar el cHDL; incluso la adición de niacina para alcanzar metas de CLDL entre 40-80 mg/dl no redujo más el RCV, por tanto la terapia de combinación ha demostrado que no proporciona un beneficio cardiovascular adicional por encima de la terapia con estatinas de forma individualizada y no se recomienda esta pauta en general ${ }^{64,77-79}$.

Si los pacientes no alcanzan los objetivos mencionados con la dosis máxima tolerada de estatinas, un objetivo terapéutico alternativo es reducir la concentración basal de $\mathrm{CLDL}$ un $50 \%$. Adicionalmente, se aconseja mantener un nivel objetivo de triglicéridos $<150 \mathrm{mg} / \mathrm{dl}(1,7 \mathrm{mmol} / \mathrm{l})$ y de $\mathrm{cHDL}$ en los varones $>40 \mathrm{mg} / \mathrm{dl}(1 \mathrm{mmol} / \mathrm{l})$ y en las mujeres $>50 \mathrm{mg} / \mathrm{dl} \quad(1,3 \mathrm{mmol} / \mathrm{l})$. Los fibratos pueden ayudar a prevenir $\mathrm{y} / \mathrm{o}$ mejorar los niveles de triglicéridos y se ha señalado un papel protector de la retinopatía diabética y de otras complicaciones microangiopáticas ${ }^{80}$. Sin embargo, no debemos olvidar que el objetivo primario del metabolismo lipídico es el tratamiento con estatinas, dirigido a mantener el nivel de CLDL dentro de los objetivos señalados. La terapia con estatinas está contraindicada en el embarazo. Cuando el paciente con DM2 precise tratamiento de combinación de una estatina asociada a un fibrato para reducir el riesgo residual atribuible a la dislipidemia aterogénica, el fibrato recomendable es fenofibrato, debido a las mayores interacciones farmacológicas del gemfibrozilo ${ }^{81,82}$. La asociación de una estatina con ezetimiba también confiere reducciones adicionales de CLDL y ha mostrado muy recientemente reducciones de episodios cardiovasculares en el estudio IMPROVE-IT 
(http://www.cardiosource.org/science-and-quality/clinicaltrials/i/improve-it.aspx?w_nav=RI).

\section{Antiagregación y otros fármacos en enfermedad cardiovascular: indicación de los antiagregantes plaquetarios, bloqueadores beta y cirugía en la enfermedad cardiovascular y diabetes}

Tanto en DM1 como en DM2 con RCV elevado (> 10\% a 10 años) el beneficio del tratamiento con ácido acetilsalicílico (75-162 mg/día) como estrategia de prevención primaria no está claramente demostrado. Se recomienda en los varones diabéticos $>50$ años o las mujeres $>60$ años que tienen al menos un FRCV mayor como historia familiar de ECV, HTA, tabaquismo, dislipidemia o microalbuminuria. No se debe recomendar ácido acetilsalicílico para la prevención primaria de la ECV en los adultos con DM2 y bajo RCV $(<5 \%$ a los 10 años) como los varones $<50$ años y las mujeres $<60$ años sin otros FRCV, ya que los efectos adversos potenciales de sangrado probablemente contrarresten el posible beneficio ${ }^{83-85}$. En la actualidad se están realizando ensayos clínicos para valorar el papel del ácido acetilsalicílico en prevención primaria ${ }^{80}$.

En los diabéticos con ECV establecida se debe indicar ácido acetilsalicílico (75-162 mg/día) como estrategia de prevención secundaria. Para los pacientes con ECV y alergia documentada al ácido acetilsalicílico se debe utilizar el clopidogrel (75 mg/día). Hasta un año después de un síndrome coronario agudo (SCA) es razonable el tratamiento combinado de ácido acetilsalicílico (75-162 mg/día) y clopidogrel (75 mg/día). Se recomienda usar durante un año un inhibidor del receptor P2Y12 para pacientes con DM2 y SCA y para los sometidos a intervención coronaria percutánea (ICP) (la duración depende del tipo de stent). Para pacientes con ICP por SCA se debe administrar preferiblemente prasugrel o ticagrelor.

En los pacientes con ECV hay que tener en cuenta el tratamiento con IECA, estatinas (si no están contraindicadas) para reducir el riesgo de episodios cardiovasculares y ácido acetilsalicílico. En los pacientes con un infarto de miocardio previo los bloqueadores beta se deben mantener por lo menos 2 años después del episodio agudo. El uso de bloqueadores beta en el postinfarto es primordial para prevenir la muerte súbita ${ }^{86}$. En la actualidad la cirugía cardiaca debe ser la opción terapéutica para la mayoría de DM2 con enfermedad multivaso conocida ${ }^{86-88}$.

\section{Diabetes y factores de riesgo cardiovascular en situaciones especiales}

\section{Enfermedad renal crónica y diabetes}

La ERC se define como la presencia durante al menos 3 meses de daño renal o $\mathrm{FG}<60 \mathrm{ml} / \mathrm{min} / 1,73 \mathrm{~m}^{2}$ o de lesiones renales estructurales (alteraciones histológicas en la biopsia renal) o funcionales (albuminuria, alteraciones en el sedimento urinario o en las pruebas de imagen) que puedan provocar potencialmente un descenso del FG. La detección precoz de los pacientes con ERC y su tratamiento es fundamental para disminuir la morbilidad cardiovascular y la velocidad de progresión de la enfermedad renal, reduciendo los costes para el sistema sanitario ${ }^{89,90}$. Se define como nefropatía diabética la afectación renal en un paciente diagnosticado de DM2 con proteinuria superior a $300 \mathrm{mg} /$ día (nefropatía incipiente si se detectan valores en el rango de $30-300$ mg/día) en ausencia de otra enfermedad renal. La nefropatía diabética se acompaña muy frecuentemente de HTA y de disminución de la función renal ${ }^{89}$.

Los objetivos actuales del diabético con ERC son: HbA1c (convencional) $<7 \%$ (NGSP/DCTT). Como se ha señalado recientemente, los objetivos de control glucémico son menos estrictos en pacientes ancianos, o con esperanza de vida limitada, elevada comorbilidad, historia previa de hipoglucemias o en pacientes con conocida DM2 de larga evolución. La metformina es la primera indicación en diabéticos, y en los últimos años ha aumentado de forma considerable la experiencia en la utilización de la misma, de tal forma que, basándose en estudios observacionales, su empleo se considera razonablemente seguro en pacientes con FG 45-60 ml/min/1,73 $\mathrm{m}^{2}$, monitorizando la función renal cada 3-6 meses; si el FG $30-45 \mathrm{ml} / \mathrm{min} / 1,73 \mathrm{~m}^{2}$ se recomienda reducir al $50 \%$ la dosis de metformina, y $\mathrm{si}$ $<30 \mathrm{ml} / \mathrm{min} / 1,73 \mathrm{~m}^{2}$ no debe utilizarse. La repaglinida es el secretagogo más indicado en ERC, pudiéndose utilizar en diálisis. Las gliptinas pueden utilizarse en ERC, son eficaces y seguras, aunque requieren ajuste de dosis según el grado de ERC, salvo la linagliptina ${ }^{89-92}$. La pioglitazona también está indicada en pacientes con ERC que no tengan alguna contraindicación al fármaco, y prestando atención a la posible retención hidrosalina que puede ser un problema en fases avanzadas de la enfermedad. La experiencia en pacientes en diálisis es muy limitada, por lo que según el documento de consenso español sería preferible evitar su prescripción en pacientes con $\mathrm{FG}<30 \mathrm{ml} / \mathrm{min}^{90}$.

Se preconiza un control de la PA a cifras $<140 / 90 \mathrm{~mm} \mathrm{Hg}$ y a veces se sugiere $<130 / 80 \mathrm{~mm} \mathrm{Hg}$ si existe albuminuria (cociente albúmina creatinina $>30 \mathrm{mg} / \mathrm{g}$ ), con tendencia clínica a ir individualizándose el tratamiento a fin de evitar hipotensiones. Se debe disminuir la albuminuria $<300 \mathrm{mg} / \mathrm{g}$ $\mathrm{Cr}$, mediante la utilización de inhibidores del sistema reninaangiotensina-aldosterona (IECA, ARA ॥), al ser considerada la microalbuminuria como un marcador de RCV independiente. El objetivo lipídico en pacientes con ERC y la decisión de usar estatinas se hará tras la valoración individualizada del RCV. En general, en prevención primaria se recomienda tratar a los pacientes con ERC (estadios 3-5 no en diálisis) con estatinas o estatina/ezetimiba. Existen estatinas que no requieren ajuste como atorvastatina y fluvastatina, otras que precisan reducir dosis como son la simvastatina y pravastatina, y otras que no se aconsejan en ERC avanzada ${ }^{90,91}$. La ezetimiba no requiere ajuste de dosis y su complemento terapéutico es de eficacia clínica contrastada. El tratamiento de la hipertrigliceridemia en la ERC debe basarse en cambios en el estilo de vida. No se recomiendan fibratos para disminuir el riesgo cardiovascular y se desaconseja su uso si el $\mathrm{FG}<15 \mathrm{ml}^{90}$. Los fibratos y los ácidos grasos $\omega-3$ podrían ser considerados en aquellos pacientes con niveles marcadamente elevados de triglicéridos en ayunas (>500 mg/dl) para evitar el riesgo de pancreatitis ${ }^{82,93}$. La guía KDIGO aconseja el uso de antiagregantes en la ERC, y por tanto estarían 
indicados en el diabético siempre y cuando la PA esté controlada $<140 / 90 \mathrm{~mm} \mathrm{Hg}^{94}$.

\section{Diabetes en el anciano}

La diabetes en el anciano suele seguir un curso asintomático y, por otro lado, su expresión clínica es a menudo insidiosa y atípica. El paciente anciano con diabetes presenta ciertas particularidades como la presencia de una elevada heterogeneidad clínica, deterioro cognitivo, depresión o caídas y un mayor riesgo de morbimortalidad, entre otras, lo que condiciona el diagnóstico y abordaje de la enfermedad. Los ancianos con diabetes tienen más carga de comorbilidad que los no diabéticos, y también presentan más riesgo de depresión y de incapacidad funcional ${ }^{95}$.

No existe acuerdo entre las diferentes guías en cuanto al objetivo de $\mathrm{HbA} 1 \mathrm{c}$ en los pacientes ancianos, oscilando los niveles de HbA1c entre el $7 \%$ y el $9 \%{ }^{96}$. Así, el European Diabetes Working Party for Older People $2011^{96}$ aconseja un objetivo de HbA1c del 7-7,5\% para pacientes ancianos sin complicaciones y del 7,6-8,5\% para pacientes ancianos frágiles. Recientemente, la ADA/EASD recomienda que los objetivos glucémicos sean menos ambiciosos en ancianos con expectativa de vida corta, elevada morbilidad, polimedicación o alto riesgo de hipoglucemias, considerando aceptable una HbA1c entre el 7,6 y el 8,5\% ${ }^{95}$. Es importante el cribado nutricional con el fin de descartar el riesgo de desnutrición que favorece el desarrollo de la sarcopenia, una condición que se asocia a deterioro funcional, riesgo de caídas y de institucionalización en ancianos ${ }^{16}$.

La metformina sigue siendo la primera opción terapéutica, las sulfonilureas son fármacos baratos y con amplia experiencia de uso. Su mayor inconveniente son las hipoglucemias, especialmente la glibenclamida, cuyo uso está desaconsejado en el anciano, siendo preferible en caso de precisar la indicación de sulfonilureas el empleo de gliclazida o glimepirida ${ }^{97}$. Los inhibidores de la dipeptidil peptidasa-4 son bien tolerados y no favorecen la hipoglucemia en los pacientes de riesgo. Los análogos de insulina tienen menos riesgo de hipoglucemia que la insulina humana ${ }^{98}$.

El control global de los FRCV es importante en los pacientes ancianos con diabetes, y de hecho proporciona una mayor reducción de la morbimortalidad que el propio control glucémico. El tratamiento antihipertensivo produce beneficios, incluso en ancianos de edad muy avanzada. En los ancianos frágiles se aceptan cifras de $150 / 90 \mathrm{~mm} \mathrm{Hg}$, debiéndose evitar niveles $<120 / 70 \mathrm{~mm} \mathrm{Hg}^{95}$. Igualmente, existen evidencias sólidas de los beneficios de la terapia con estatinas y otros hipolipidemiantes no estatinas, como los inhibidores de la absorción de ácidos biliares (colesevelam) poco utilizados en nuestro medio ${ }^{98}$, por su coste más elevado y buena tolerancia de la ezetimiba. En el anciano los antiagregantes están indicados en prevención secundaria, mientras que en prevención primaria su uso es más controvertido y debería individualizarse.

\section{Otros factores de riesgo cardiovascular emergentes}

La alteración del sueño reparador, las alteraciones de los genes reguladores del ritmo circadiano, así como la esteatosis hepática se asocian al riesgo de ECV y de DM2. El nivel sociocultural es otro FRCV al que cada día se le concede más peso en el desarrollo de la diabetes y RVC. Los factores psicosociales, así como el bajo nivel socioeconómico, el aislamiento social, la depresión u hostilidad y el estrés laboral o familiar, además de asociarse a un mayor RCV, empeoran el pronóstico de los pacientes con cardiopatía isquémica y dificultan significativamente el control de los FRCV. Es de destacar el papel de la vitamina D, la insulinorresistencia y la microbiota intestinal, tanto en el desarrollo de la diabetes como de su RCV $99-104$. Por último, mencionar el papel de la homocisteinemia por su efecto trombogénico. No debemos olvidar que el uso de metformina puede disminuir los niveles de vitamina $B_{12}$ y reducir la reciclación de la homocisteína a metionina, elevando los niveles plasmáticos de homocisteína ${ }^{104}$.

\section{Conclusiones}

El control de los FRCV previene el desarrollo de la ECV y/o la retrasa en el tiempo. Sin embargo, los estudios epidemiológicos muestran de forma contundente un deficiente control de los mismos en los pacientes con DM2. Es por tanto imperativo mejorar el control cardiometabólico de los pacientes con DM2 y alcanzar los objetivos terapéuticos del mayor número posible de FRCV. Es aconsejable utilizar el procedimiento diagnóstico más asequible para cada paciente, así como individualizar la estrategia terapéutica. La dieta mediterránea y el ejercicio aeróbico son beneficiosos en el diabético, facilitando el control de los FRCV y la consecución de los objetivos terapéuticos; en función de las evidencias disponibles no son necesarios los suplementos de antioxidantes ni vitaminas si no existen deficiencias. La CB parece ser cada vez más eficiente, disminuyendo a corto y largo plazo las comorbilidades y complicaciones de la diabetes, así como revirtiendo la enfermedad en un número significativo de pacientes obesos con DM2. Se debe conseguir la mayor adherencia de los pacientes al tratamiento, así como una mayor implicación y objetivos más realistas de las guías, individualizando el tratamiento y sus objetivos y adaptándolos al entorno del paciente.

\section{Bibliografía}

1. Diabetes mellitus y riesgo cardiovascular. Recomendaciones del Grupo de trabajo diabetes mellitus y enfermedad cardiovascular de la Sociedad Española de Diabetes, 2009. Av Diabeto. 2009;25:449-54.

2. Grupo de trabajo diabetes mellitus y enfermedad cardiovascular de la Sociedad Española de Diabetes. Diabetes Mellitus and cardiovascular risk. Recommendations of the working group of diabetes mellitus and cardiovascular disease of the Spanish Diabetes Society 2009. Endocrinol Nutr. 2010;57:220-6.

3. Standards of medical care in diabetes 2014 ADA Clinical Practice Recommendations. Diabetes Care. 2014;37(Suppl1):s14s80.

4. Goff DC Jr, Lloyd-Jones DM, Bennett G, Coady S, D’Agostino RB Sr, Gibbons R, et al. 2013 ACC/AHA guideline on the assessment of cardiovascular risk. J Am Coll Cardiol. 2014;63:2935-59.

5. Arrieta F, Rubio-Terrés C, Rubio-Rodríguez D, Magaña A, Piñera $M$, Iglesias $P$, et al. Study group on diabetes estimation of the economic and health impact of complications of type 2 
diabetes mellitus in the autonomous community of Madrid (Spain). Endocrinol Nutr. 2014;61:193-201.

6. Pantoja T, Strain H, Valenzuela L. Guías de práctica clínica en atención primaria: una evaluación crítica. Rev Méd Chile. 2007; 135:1282-90.

7. Maiques A, Villar F, Brotons C, Torcal J, Orozco D, Navarro $\mathrm{J}$, et al. Recomendaciones preventivas cardiovasculares. Aten Primaria. 2007;39 Suppl 3:15-26.

8. Arrieta F, Salinero M, Piñera M, Botella-Carretero JI, Iglesias P, Abanadas JC, et al. Estudio descriptivo de la evolución clínicoasistencial de la población con diabetes tipo 2 en la Comunidad de Madrid. Estudio de seguimiento diabético tipo 2 (ESD2). Av Diabetol. 2011;27:53-6.

9. Roca-Rodríguez MM, Carral-San Laureano F, Baena-Nieto G, Aguilar-Diosdado M. Evaluación del grado de consecución de objetivos de control metabólico en pacientes con diabetes mellitus de tipo 2. Endocrinol Nutr. 2010;57:434-9.

10. Unick JL, Beavers D, Jakicic JM, Kitabchi AE, Knowler WC, Wadden TA, et al., Look AHEAD research group. Effectiveness of lifestyle interventions for individuals with severe obesity and type 2 diabetes: Results from the Look AHEAD trial. Diabetes Care. 2011;34:2152-7.

11. Wing R, Lang W, Wadden T, Safford M, Knowler W, Breton A, et al., The Look AHEAD research group. Benefits of modest weight loss in improving cardiovascular risk factors in overweight and obese individuals with type 2 diabetes. Diabetes Care. 2012;34:1481-6.

12. Wing RR, Bolin P, Brancati FL, Bray GA, Clark JM, Coday M, et al., Look AHEAD research group. Cardiovascular effects of intensive lifestyle intervention in type 2 diabetes. N Engl J Med. 2013;369:145-54.

13. Look AHEAD Research Group. Eight-year weight losses with an intensive lifestyle intervention: the look AHEAD study. Obesity (Silver Spring). 2014;22:5-13.

14. Stratton RJ, Green CJ, Elia M. Disease-related malnutrition: An evidence-based approach to treatment. Oxford, United Kingdom: CABI Publishing; 2003.

15. Cawood AL, Elia M, Sharp SK, Stratton RJ. Malnutrition selfscreening by using MUST in hospital outpatients: Validity, reliability, and ease of use. Am J Clin Nutr. 2012;96:1000-7.

16. De Ulíbarri Pérez JI, Fernández G, Rodríguez Salvanés F, Díaz López AM. Nutritional screening; control of clinical undernutrition with analytical parameters. Nutr Hosp. 2014;29: 797-811.

17. Estruch R, Ros E, Salas-Salvadó J, Covas MI, Corella D, Arós F, et al., The PREDIMED Study Investigators. Primary prevention of cardiovascular disease with a mediterranean diet. N Engl J Med. 2013;368:1279-90.

18. De Lorgeril M, Salen P, Martin JL, Monjaud I, Delaye J, Mamelle N. Mediterranean diet, traditional risk factors, and the rate of cardiovascular complications after myocardial infarction: Final report of the Lyon Diet Heart Study. Circulation. 1999;99:779-85.

19. Salas-Salvadó J, Bulló M, Estruch R, Ros E, Covas MI, IbarrolaJurado $\mathrm{N}$, et al. Prevention of diabetes with mediterranean diets: A subgroup analysis of a randomized trial. Ann Intern Med. 2014;160:1-10.

20. Jenkins DJ, Kendall CW, McKeown-Eyssen G, Josse RG, Silverberg J, Booth GL, et al. Effect of a low-glycemic index or a high-cereal fiber diet on type 2 diabetes: A randomized trial. JAMA. 2008;300:2742-53.

21. Yu D, Shu XO, Li H, Xiang YB, Yang G, Gao YT, et al. Dietary carbohydrates, refined grains, glycemic load, and risk of coronary heart disease in Chinese adults. Am J Epidemiol. 2013;178:1542-9.

22. Juanola-Falgarona M, Salas-Salvadó J, Ibarrola-Jurado N, Rabassa Soler A, Díaz-López A, Guasch-Ferré M, et al. Effect of the glycemic index of the diet on weight loss, modulation of satiety, inflammation, and other metabolic risk factors: A randomized controlled trial. Am J Clin Nutr. 2014;100:27-35.

23. Fletcher GF, Ades PA, Kligfield P, Arena R, Balady GJ, Bittner VA, et al. Exercise standards for testing and training: A scientific statement from the American Heart Association. Circulation. 2013;128:873-934.

24. Cordero A, Masiá MD, Galve E. Ejercicio físico y salud. Rev Esp Cardiol. 2014;67:748-53.

25. Lee IM, Shiroma EJ, Lobelo F, Puska P, Blair SN, Katzmarzk PT. Effect of physical inactivity on major non-communicable diseases worldwide: An analysis of burden of disease and life expectancy. Lancet. 2012;380:219-29.

26. Nelson ME, Rejeski J, Blair SN, Duncan PW, Judge JO, King AC, et al. Physical activity and public health in older adults: Recommendation from the American College of Sports Medicine and the American Heart Association. Circulation. 2007;116:1094-105.

27. Dunstan DW, Daly RM, Owen N. High-intensity resistance training improves glycemic control in older patients with type 2-diabetes. Diabetes Care. 2002;25:1729-36.

28. Colberg SR, Sigal RJ, Fernhall B, Regensteiner JG, Blissmer BJ, Rubin RR, et al., American College of Sports Medicine; American Diabetes Association. Exercise and type 2 diabetes: The American College of Sports Medicine and the American Diabetes Association: Joint position statement. Diabetes Care. 2010;33:e147-67.

29. Sigal RJ, Kenny GP, Boulé NG, Wells GA, Prud'homme D, Fortier $M$, et al. Effects of aerobic training, resistance training, or both on glycemic control in type 2 diabetes: A randomized trial. Ann Intern Med. 2007;147:357-69.

30. Umpierrez D, Ribeiro PA, Kramer CK, Leitão CB, Zucatti AT, Azevedo MJ, et al. Physical activity advice only or structured exercise training and association with $\mathrm{HbA1c}$ levels in type 2 diabetes: A systematic review and meta-analysis. JAMA. 2011;305:1790-9.

31. Qin R, Chen T, Lou Q, Yu D. Excess risk of mortality and cardiovascular events associated with smoking hmong patients with diabetes meta-analysis of observational prospective studies. Int J Cardiol. 2013;167:342-50.

32. Sattar N. Advances in managing type 2 diabetes: challenging old paradigms and developing new ones. F1000 Prime Rep. 2014. Jun 2;6:42. doi: 10.12703/P 6-42. eCollection 2014.

33. Salas-Salvadó J, Rubio MA, Barbany M, Moreno B, Grupo colaborativo de la SEEDO. Consenso SEEDO 2007 para la evaluación del sobrepeso y la obesidad y el establecimiento de criterios de intervención terapéutica. Med Clin (Barc). 2007;128:184-96.

34. McNeely MJ, Shofer JB, Leonetti DL, Fujimoto WY, Boyko EJ. Associations among visceral fat, all-cause mortality, and obesity-related mortality in Japanese Americans. Diabetes Care. 2012;35:296-8.

35. Piñera MJ, Arrieta FJ, Alcaraz-Cebrián F, Botella-Carretero JI, Calañas A, Balsa JA, et al. Influencia de la pérdida de peso en la evolución clínica, metabólica y psicológica de los pacientes con sobrepeso u obesidad. Nutr Hosp. 2012;27:1480-8.

36. Rubio MA, Monereo S, Lecube A, Resa J, Masdevall C, de la Cruz $\mathrm{F}$, et al. Posicionamiento de las sociedades SEEN-SECO-SEEDOSED sobre la cirugía metabólica en la diabetes mellitus tipo2. Endocrinol Nutr. 2013;60:547-8.

37. Ngiam KY, Lee WJ, Lee YC, Cheng A. Efficacy of metabolic surgery on $\mathrm{HbA} 1 \mathrm{c}$ decrease in type 2 diabetes mellitus patients with $\mathrm{BMI}<35 \mathrm{~kg} / \mathrm{m}^{2}-$ a review. Obes Surg. 2014;24:148-58.

38. Buchwald H, Oien DM. Metabolic/bariatric surgery worldwide 2011. Obes Surg. 2013;23:427-36.

39. Lee YC, Lee WJ, Liew PL. Predictors of remission of type 2 diabetes mellitus in obese patients after gastrointestinal surgery. Obes Res Clin Pract. 2013;7:e494-500.

40. Still CWG, Benotti P, Petrick AT, Gabrielsen J, Strodel WE, Ibele A, et al. Preoperative prediction of type 2 diabetes remission 
after Roux-en-Y gastric bypass surgery: A retrospective cohort study. Lancet Diabetes Endocrinol. 2014;2:3845.

41. Ikramuddin S1, Korner J, Lee WJ, Connett JE, Inabnet WB, Billington $\mathrm{CJ}$, et al. Roux-en-Y gastric bypass vs intensive medical management for the control of type 2 diabetes, hypertension, and hyperlipidemia: The diabetes surgery study randomized clinical trial. JAMA. 2013;309:2240-9.

42. Mas-Lorenzo A, Benaiges D, Flores-Le-Roux JA, Pedro-Botet J, Ramon JM, Parri A, et al., On behalf of the Obemar Group. Impact of different criteria on type 2 diabetes remission rate after bariatric surgery. Obes Surg. 2014;24:1881-7.

43. Lee WJ, Chong K, Lin YH, Wei JH, Chen SC. Laparoscopic sleeve gastrectomy versus single anastomosis (mini-) gastric bypass for the treatment of type 2 diabetes mellitus: 5-year results of a randomized trial and study of incretin effect. Obes Surg. 2014;24:1552-62.

44. Svane MS, Madsbad S. Bariatric surgery-Effects on obesity and related co-morbidities. Curr Diabetes Rev. 2014;10:208-14.

45. Cohen R, Caravatto PP, Petry T, Cummings D. Role of metabolic surgery in less obese or non-obese subjects with type 2 diabetes: Influence over cardiovascular events. Curr Atheroscler Rep. 2013;15:355-63.

46. Balsa JA, Botella-Carretero JI, Gómez-Martín JM, Peromingo R, Arrieta F, Santiuste C, et al. Copper and zinc serum levels after derivative bariatric surgery: Differences between roux en-Y gastric bypass and biliopancreatic diversion. Obes Surg. 2011;21:744-50.

47. García BF, Peromingo R, Galindo J, Arrieta F, Sánchez J, Vázquez $\mathrm{C}$, et al. Case report subtotal pancreatectomy as treatment for severe hypoglycemia after gastric bypass. Endocrinol Nutr. 2014;61:391-3.

48. Hoerger TJ, Zhang P, Segel JE, Kahn HS, Barker LE, Couper S. Cost-effectiveness of bariatric surgery for severely obese adults with diabetes. Diabetes Care. 2010;33:1193-9.

49. Keating CL, Peeters A, Swinburn BA, Carter R, Moodie ML. Pharmaceutical utilisation and costs before and after bariatric surgery. Int J Obes (Lond). 2013;37:1467-72.

50. Makary MA, Clark JM, Shore AD, Magnuson TH, Richards T, Bass $\mathrm{EB}$, et al. Medication utilization and annual health care costs in patients with type 2 diabetes mellitus before and after bariatric surgery. Arch Surg. 2010;145:726-31.

51. Malkani S1, Mordes JP. Implications of using hemoglobin $\mathrm{A} 1 \mathrm{C}$ for diagnosing diabetes mellitus. Am J Med. 2011;124:395-401.

52. Christensen DL, Witte DR, Kaduka L, Jørgensen ME, BorchJohnsen K, Mohan V, et al. Moving to an A1C-based diagnosis of diabetes has a different impact on prevalence in different ethnic groups. Diabetes Care. 2010;33:580-2.

53. Olson DE, Rhee MK, Herrick K, Ziemer DC, Twombly JG, Phillips LS. Screening for diabetes and pre-diabetes with proposed A1C-based diagnostic criteria. Diabetes Care. 2010;33:2184-9.

54. UK Prospective Diabetes Study. Intensive blood glucose control with sulfonylureas or insulin compared with conventional treatment and risk of complications in patientes with type 2 diabetes (UKPDS 33). Lancet. 1998;352:837-53.

55. Gaude P, Vedel P, Larsen N, Jensen GVH, Parving H, Pedersen 0 . Multifactorial interventions and cardiovascular disease in patients with type 2 diabetes. N Engl J Med. 2003;348:383-93.

56. Patel A, MacMahon S, Chalmers J, Neal B, Billot L, Woodward M, et al. ADVANCE Collaborative Group, intensive blood glucose control and vascular outcomes in patients with type 2 diabetes. N Engl J Med. 2008;358:2560-72.

57. Vaag AA. Glycemic control and prevention of microvascular and macrovascular disease in the Steno 2 study. Endocr Pract. 2006;12 Suppl 1:89-92.

58. Inzucchi SE, Bergenstal RM, Buse JB, Diamant M, Ferrannini E, Nauck M, et al., European Association for the Study of Diabetes (EASD). Management of hyperglycemia in type 2 diabetes: A patient-centered approach: position statement of the American Diabetes Association (ADA) and the European Association for the Study of Diabetes (EASD). Diabetes Care. 2012;35:1364-79.

59. Qaseem A, Humphrey LL, Sweet DE, Starkey M, Shekelle P, Clinical guidelines Committee of the American College of Physicians. Oral pharmacologic treatment of type 2 diabetes mellitus: A clinical practice guideline from the American College of Physicians. Ann Intern Med. 2012;156:218-31.

60. Wilt TJ, Qaseem A. Implementing high-value, cost-conscious diabetes mellitus care through the use of low-cost medications and less-intensive glycemic control target. Arch Intern Med. 2012;172:1610-1.

61. Preiss D. Statin therapy and new-onset diabetes: A cause for concern. Maturitas. 2012;72:95-6.

62. Sattar NA, Ginsberg H, Ray K, Chapman MJ, Arca M, Averna $M$, et al. The use of statins in people at risk of developing diabetes mellitus: Evidence and guidance for clinical practice. Atheroscler Suppl. 2014;15:1-15.

63. Abdul-Ghani MA, Tripathy D, DeFronzo RA. Contributions of beta-cell dysfunction and insulin resistance to the pathogenesis of impaired glucose tolerance and impaired fasting glucose. Diabetes Care. 2006;29:1130-9.

64. Rydén L, Grant PJ, Anker SD, Berne C, Cosentino F, Danchin $\mathrm{N}$, et al., Task Force on diabetes, pre-diabetes, and cardiovascular diseases of the European Society of Cardiology (ESC); European Association for the Study of Diabetes (EASD). ESC guidelines on diabetes, pre-diabetes, and cardiovascular diseases developed in collaboration with the EASD-summary. Diab Vasc Dis Res. 2014;11:133-73.

65. JBS3 Board Joint British Societies' consensus recommendations for the prevention of cardiovascular disease (JBS3). Heart. 2014;100 Suppl 2:ii1-ii67. DOI: 10.1136/heartjnl-2014-305693.

66. Mancia G, Fagard R, Narkiewicz K, Redon J, Zanchetti A, Bohm $\mathrm{M}$, et al. ESH/ESC guidelines for the management of arterial hypertension; The task force for the management of arterial hypertension of the European Society of Hypertension (ESH) and of the European Society of Cardiology (ESC). J Hypertens. 2013;31:1281-357.

67. Lopez-Jaramillo P, Sanchez R, Diaz M, Cobos L, Bryce A, ParraCarrillo JZ, et al., On behalf of the Latin America expert Group. Latin American consensus on hypertension in patients with diabetes type 2 and metabolic syndrome. J Hypertens. 2013;31:223-38.

68. Rossen NB, Knudsen ST, Fleischer J, Hvas AM, Ebbehøj E, Poulsen PL, et al. Targeting nocturnal hypertension in type 2 diabetes mellitus. Hypertension. 2014;64:1080-7.

69. Barnes TL, Crandell JL, Bell RA, Mayer-Davis EJ, Dabelea D, Liese AD. Change in DASH diet score and cardiovascular risk factors in youth with type 1 and type 2 diabetes mellitus: The SEARCH for diabetes in youth study. Nutr Diabetes. 2013; 3:e91.

70. Maggioni AP, Anker SD, Dahlström U, Filippatos G, Ponikowski $P$, Zannad F, et al., Heart failure association of the ESC. Are hospitalized or ambulatory patients with heart failure treated in accordance with European Society of Cardiology guidelines? Evidence from 12,440 patients of the ESC heart failure longterm Registry. Eur J Heart Fail. 2013;15:1173-84.

71. Kolata G. Risk calculator for cholesterol appears flawed. New York Times. 17 November 2013 [consultado 9 Ene 2014]. Disponible en: www.nytimes.com/2013/11/18/health/ risk-calculator-for-cholesterol-appears-flawed.html?_r=0

72. Maki KC, Ridker PM, Brown WV, Grundy SM, Sattar N. An assessment by the Statin diabetes safety task force: 2014 update. J Clin Lipidol. 2014;8 3 Suppl:S17-29.

73. Ray K. Statin diabetogenicity: Guidance for clinicians. Cardiovasc Diabetol. 2013;12 Suppl 1:S3, http://dx.doi.org/ 10.1186/1475-2840-12-S1-S3. 
74. Downs J, Good C. New cholesterol guidelines: Has God ot finally arrived? Ann Intern Med. 2014;160:354-5.

75. Bolli P. Treatment of dyslipidemia: The problem of reaching the goal. Atherosclerosis. 2014;236:142-3.

76. Carter AA, Gomes T, Camacho X, Juurlink DN, Shah BR, Mamdani MM. Risk of incident diabetes among patients treated with statins: Population based study. BMJ. 2013;346:f2610.

77. National Cholesterol Education Program (NCEP) Expert Panel on Detection, Evaluation, and Treatment of High Blood Cholesterol in Adults (Adult Treatment Panel III). Third report of the National Cholesterol Education Program (NCEP) Expert Panel on Detection, Evaluation, and Treatment of High Blood Cholesterol in Adults (Adult Treatment Panel III) final report. Circulation. 2002;106:3143-421.

78. Blazing MA, Giugliano RP, Cannon CP, Musliner TA, Tershakovec AM, White JA, et al. Evaluating cardiovascular event reduction with ezetimibe as an adjunct to simvastatin in 18,144 patients after acute coronary syndromes: Final baseline characteristics of the IMPROVE-IT study population. Am Heart J. 2014; $168: 205-12$.

79. Stone NJ, Robinson JG, Lichtenstein AH, Goff DC Jr, LloydJones DM, Smith SC Jr, et al. 2013 ACC/AHA Cholesterol Guideline Panel. Treatment of blood cholesterol to reduce atherosclerotic cardiovascular disease risk in adults: synopsis of the 2013 American College of Cardiology/American Heart Association cholesterol guideline. Ann Intern Med. 2014;160:339-43.

80. Agouridis AP, Rizos CV, Elisaf MS, Filippatos TD. Does combination therapy with statins and fibrates prevent cardiovascular disease in diabetic patients with atherogenic mixed dyslipidemia. Rev Diabet Stud. 2013;10:171-90.

81. Simó R, Roy S, Behar-Cohen F, Keech A, Mitchell P, Wong TY. Fenofibrate: A new treatment for diabetic retinopathy. Molecular mechanisms and future perspectives. Curr Med Chem. 2013;20:3258-66.

82. Tenenbaum A, Fisman EZ. Fibrates are an essential part of modern anti-dyslipidemic arsenal: Spotlight on atherogenic dyslipidemia and residual risk reduction. Cardiovasc Diabetol. 2012;11:125. doi: 10.1186/1475-2840-11-125.

83. Lorber D. Importance of cardiovascular disease risk management in patients with type 2 diabetes mellitus. Diabetes Metab Syndr Obes. 2014;23:169-83.

84. Pignone M, Alberts MJ, Colwell JA, Cushman M, Inzucchi SE, Mukherjee D, et al. Aspirin for primary prevention of cardiovascular events in people with diabetes: A position statement of the American Diabetes Association, a scientific statement of the American Heart Association, and an expert consensus document of the American College of Cardiology Foundation. Diabetes Care. 2010;33:1395-402.

85. Lattanzio S, Santilli F, Liani R, Vazzana N, Ueland T, di Fulvio $P$, et al. Circulating dickkopf-1 in diabetes mellitus: Association with platelet activation and effects of improved metabolic control and low-dose aspirin. J Am Heart Assoc. 2014;3:e001000, http://dx.doi.org/ 10.1161/JAHA.114.001000.

86. Sinclair AJ, Paolisso G, Castro M, Bourdel-Marchasson I, Gadsby R, Rodriguez Mañas L, European diabetes working party for older people. European diabetes working party for older people 2011 clinical guidelines for type 2 diabetes mellitus executive summary. Diabetes Metab. 2011;37 Suppl 3:S27-38.
87. Burgess SN, Mussap CJ, French JK. Management of acute coronary syndromes in patients with diabetes: Implications of the FREEDOM trial. Clin Ther. 2013;35:1069-75.

88. Deb S, Wijeysundera HC, Ko DT, Tsubota H, Hill S, Fremes SE. Coronary artery bypass graft surgery vs percutaneous interventions in coronary revascularization: A systematic review. JAMA. 2013;310:2086-95.

89. Alcázar R, Portolés JM, Egocheaga I, Lobos JM, Rosado J, Arrieta F, et al. Recomendaciones sobre la detección, seguimiento y criterios de derivación de la enfermedad renal crónica en el ámbito de la Comunidad de Madrid. Médicos de familia. 2011;13:9-16.

90. Gómez-Huelgas R, Martínez-Castelao A, Artola S, Górriz JL, Menéndez E. Grupo de tabajo para el documento de consenso sobre el tratamiento de la diabetes tipo 2 en el paciente con enfermedad renal crónica. Nefrologia. 2014;34:34-45.

91. Iglesias P, Heras M, Díez JJ. Diabetes mellitus y enfermedad renal en el anciano. Nefrologia. 2014;34:285-92.

92. Lewis G, Maxwell AP. Risk factor control is key in diabetic nephropathy. Practitioner. 2014;258:13-7.

93. Davidson MH, Phillips AK, Kling D, Maki KC. Addition of omega-3 carboxylic acids to statin therapy in patients with persistent hypertriglyceridemia. Expert Rev Cardiovasc Ther. 2014;4:1-10.

94. U.S. renal data system: USRDS 2013 annual data report: Atlas of chronic kidney disease and end-stage renal disease in the United States. Bethesda, MD: National Institutes of Health, National Institute of Diabetes and Digestive and Kidney Diseases; 2013.

95. Gómez Huelgas R, Díez-Espino J, Formiga F, Lafita J, Rodríguez L, González-Sarmiento E, et al., en nombre del Grupo de trabajo para el documento de consenso sobre el tratamiento de la diabetes tipo 2 en el anciano. Tratamiento de la diabetes tipo 2 en el paciente anciano. Med Clin (Barc). 2014;142:89-90.

96. Paolisso G, Sinclair AJ. Effective clinical practice for older people with type 2 diabetes. Glucose regulation is important but other strategies also need to be included. J Nutr Health Aging. 2010;14:3-4.

97. Arrieta FJ, Calvo MJ, Peréz A, Saavedra P, Cordobés FJ, Cabral R, et al. Prevalencia y consumo de medicación en diabetes mellitus en la comunidad de Madrid. Rev Clin Esp. 2006;206:117-21.

98. Taylor JR, Campbell KM. Insulin analogs for the management of type 2 diabetes. Am J Health Syst Pharm. 2013;70:320-34.

99. Gavin JR, Jones M, Ford D, Truitt K. Safety, efficacy of colsevelam HCL in treatment of elderly patients. Drugs Aging. 2014;31:461-70.

100. Nasri H, Behradmanesh S, Ahmadi A, Rafieian-Kopaei M. Impact of oral vitamin $D$ (cholecalciferol) replacement therapy on blood pressure in type 2 diabetes patients; a randomized, double-blind, placebo controlled clinical trial. J Nephropathol. 2014;3:29-33.

101. Nubiola A, Remolins I. Dieta hipoglucídica en el tratamiento de la diabetes tipo 2. Av Diabetol. 2012;28:131-5.

102. Lee P. PTH-vitamin D-glycemia interactions reloaded. Diabetes. 2014;63:3593-5.

103. Kassi E, Adamopoulos C, Basdra EK, Papavassiliou AG. Role of vitamin D in atherosclerosis. Circulation. 2013;128:2517-31.

104. Llanes R, Arrieta F. Diabetes y vitamina $B_{12}$ : una relación de riesgo. Diabetes News. 2014;1:32-4. 